\section{Uncertainty and Diversity in the Methods Defining Sarcopenia and Sarcopenic Obesity}

\author{
Kyoung Min Kim* \\ Division of Endocrinology and Metabolism, Department of Internal Medicine, Seoul National University Bundang \\ Hospital, Seoul National University College of Medicine, Seongnam, Korea
}

\author{
*Corresponding author \\ Kyoung Min Kim \\ https://orcid.org/0000-0001-8150-0266 \\ Division of Endocrinology and \\ Metabolism, Department of Internal \\ Medicine, Seoul National University \\ Bundang Hospital, Seoul National \\ University College of Medicine, 82 \\ Gumi-ro 173beon-gil, Bundang-gu, \\ Seongnam 13620, Korea \\ Tel: +82-31-787-7047 \\ Fax: +82-31-787-4050 \\ E-mail:kyoungmin02@gmail.com
}

As humans become older, muscle mass declines and fat mass increases; these are typical body compositional changes associated with aging. ${ }^{1}$ A reduction in muscle mass and physical activity reduces total energy expenditure in elderly people and leads to increased fat mass, characterized mainly by the accumulation of abdominal fat. ${ }^{2}$ On the other hand, increases in fat mass lead to increased secretion of a number of proinflammatory cytokines, and this accelerated inflammatory process could affect muscle losses. ${ }^{3}$ In other words, these two phenomena, loss of muscle mass and increase in fat mass, are strongly connected to each other during the aging process from a pathophysiologic point of view.

In these age-related body compositional changes, sarcopenia is defined as a condition characterized by considerable loss of muscle mass and strength, decreased physical performance, and consequent frailty in the elderly. Muscles play various important roles in the human body. Moreover, fat mass has negative effects on health status. Therefore, sarcopenia combined with obesity result in many adverse health outcomes including functional and metabolic derangements, and is becoming a major health issue in the elderly. ${ }^{4}$ As a result, recent studies have been increasingly focusing on the clinical consequences of sarcopenia or sarcopenic obesity on fractures, diabetes, cerebrovascular diseases and mortality. ${ }^{5}$
On the other hand, studies investigating the effect of sarcopenia on osteoarthritis are lacking. Skeletal muscles are essential for locomotion and mobility. Therefore, it is conceivable that sarcopenia could be significantly associated with osteoarthritis, including knee osteoarthritis or lumbar spondylitis. Jin et al. ${ }^{6}$ investigated the association between body composition characteristics that were divided into four categories: normal, sarcopenia, sarcopenic obesity, and obesity, and osteoarthritis using data from the Korea National Health and Nutrition Examination Survey (KNHANES). This is an interesting topic and an important issue. As a result, the authors ${ }^{6}$ showed that obesity with or without sarcopenia was significantly associated with knee osteoarthritis in female subjects, whereas only the sarcopenic obesity group showed a significant association with knee osteoarthritis in male subjects before further adjusting for waist circumference. Moreover, the authors ${ }^{6}$ did not observe any significant association between body composition and lumbar spondylitis regardless of gender. Considering the crucial roles of muscle in physical activity and locomotive function, these observed associations were weaker than those plausibly expected. In this respect, the question of the suitability of the criteria used for sarcopenia or obesity should be raised as Jin et al. ${ }^{6}$ suggested in their conclusion. 
Several mathematical methods using muscle values derived from dual X-ray absorptiometry or bioelectrical impedance analysis have been proposed to quantify relative muscle mass from the absolute values of regional muscle masses. Appendicular lean mass (ALM), which is the sum of the muscle mass of both arms and legs, is generally used for the muscle mass index. However, muscle mass is fundamentally correlated with body size, indicating that subjects with a larger body size may have larger muscle mass. Therefore, when evaluating the adequacy of muscle mass, the absolute level of ALM has been used after adjusting for body size in different ways, namely using height squared (ALM/ht ${ }^{2}$ ), weight (ALM/wt), or body mass index (ALM/BMI). Among those indices, $\mathrm{ALM} / \mathrm{ht}^{2}$ was first suggested by Baumgartner et al. ${ }^{7}$ However, because this index is positively correlated with BMI, it has the limitation that subjects with a greater BMI due to a larger amount of fat are less likely to be classified as having sarcopenia. In 2002, a weight-adjusted muscle mass index was proposed by Janssen et al. ${ }^{8}$ Their skeletal muscle mass index calculated the total muscle mass adjusted for weight. This weight adjusted model was subsequently modified as ALM/wt that was used by Jin et al. ${ }^{6}$ However, more recently, another muscle mass index, the ALM/BMI index, was introduced by the Foundation for the National Institutes of Health Sarcopenia Project in 2014 and use of this index is growing. ${ }^{9}$ However, among these parameters, the most appropriate method with the highest predictive value for identifying subjects who are at higher risk of weakness and slowness still remains uncertain. Moreover, the prevalence of sarcopenia varies considerably depending on the method used..$^{10}$ Furthermore, the BMI criterion that was used to define obesity by Jin et al. $^{6}$ is also limited for classifying individuals into true higher fat mass groups. Thus, the weak associations between body composition characteristics and osteoarthritis observed in that study may have been partially influenced by the limitations of the criterion used to classify sarcopenia and obesity.

The contribution of sarcopenia and obesity to the occurrence or progression of osteoarthritis in the elderly population may be still inconclusive and further investigations are needed to focus on resolving this issue.

\section{CONFLICTS OF INTEREST}

The author declares no conflict of interest.

\section{REFERENCES}

1. Shimokata H, Ando F, Yuki A, Otsuka R. Age-related changes in skeletal muscle mass among community-dwelling Japanese: a 12-year longitudinal study. Geriatr Gerontol Int 2014;14 Suppl 1:85-92.

2. Frontera WR, Ochala J. Skeletal muscle: a brief review of structure and function. Calcif Tissue Int 2015;96:183-95.

3. Kim TN, Choi KM. Sarcopenia: definition, epidemiology, and pathophysiology. J Bone Metab 2013;20:1-10.

4. Karakelides H, Nair KS. Sarcopenia of aging and its metabolic impact. Curr Top Dev Biol 2005;68:123-48.

5. Narici MV, Maffulli N. Sarcopenia: characteristics, mechanisms and functional significance. Br Med Bull 2010;95:13959.

6. Jin WS, Choi EJ, Lee SY, Bae EJ, Lee TH, Park J. Relationships among obesity, sarcopenia, and osteoarthritis in the elderly. J Obes Metab Syndr 2017;26:36-44.

7. Baumgartner RN, Koehler KM, Gallagher D, Romero L, Heymsfield SB, Ross RR, et al. Epidemiology of sarcopenia among the elderly in New Mexico. Am J Epidemiol 1998;147: 755-63.

8. Janssen I, Heymsfield SB, Ross R. Low relative skeletal muscle mass (sarcopenia) in older persons is associated with functional impairment and physical disability. J Am Geriatr Soc 2002; 50:889-96.

9. Studenski SA, Peters KW, Alley DE, Cawthon PM, McLean RR, Harris TB, et al. The FNIH sarcopenia project: rationale, study description, conference recommendations, and final estimates. J Gerontol A Biol Sci Med Sci 2014;69:547-58.

10. Kim KM, Jang HC, Lim S. Differences among skeletal muscle mass indices derived from height-, weight-, and body mass index-adjusted models in assessing sarcopenia. Korean J Intern Med 2016;31:643-50. 